\title{
DIAGONALIZATION OF A SELF-ADJOINT OPERATOR ACTING ON A HILBERT MODULE
}

\section{PARFENY P. SAWOROTNOW}

\author{
Department of Mathematics \\ The Catholic University of America \\ Washington, D.C. 20064 \\ (Received January 18, 1984)
}

ABSTRACT. For each bounded self-adjoint operator $T$ on a Hilbert module $H$ over an $\mathrm{H}^{*}$-algebra $A$ there exists a locally compact space $M$ and a certain $A$-valued measure $\mu$ such that $\mathrm{H}$ is isomorphic to $\mathrm{L}^{2}(\mu) \mathrm{A}$ and $\mathrm{T}$ corresponds to a multiplication with a continuous function. There is a similar result for a commuting family of normal operators. A consequence for this result is a representation theorem for generalized stationary processes.

KEY WORDS AND PHRASES. H*-algebra, Hilbert module, A-linear operator. 1980 MATHEMATICS SUBJECT CLASSIFICATION CODE. PRIMARY: 46H25. SECONDARY: 46K15, 47BI0, $47 \mathrm{~A} 67,46 \mathrm{G} 10,60 \mathrm{G} 10$.

\section{l. INTRODUCTION.}

The diagonalization theorem states that for each bounded self-adjoint linear operator $\mathrm{T}$ acting on a $\mathrm{Hilbert}$ space $\mathrm{H}$ there exists a measure space $(\mathrm{S}, \boldsymbol{\mu})$ and a real valued measurable function $h(s)$ such that $H$ is isomorphic to $L^{2}(S, \mu)$ and $T$ corresponds to the multiplication with $h(s)$. Furthermore, the space $(S, \mu)$ could be selected in such a way that there is a Hausdorff topology on $S$ with respect to which $h(s)$ is continuous, $S$ is locally compact and which makes $\mu$ a regular Borel measure. In this note we shall give a suitable generalization of this fact.

The situation is somewhat more complex in our case. The space $\mathrm{L}^{2}(\mathrm{~S}, \mu)$ needs to be replaced by the tensor product $\mathrm{L}^{2}(\mu) \otimes \mathrm{A}$, which is less manageable. This space is properly defined below.

2. PRELIMINARIES.

Let $A$ be a proper $H^{*-a l g e b r a ~(A m b r o s e ~[1]) ~ a n d ~ l e t ~} r A=\{x y \mid x, y+A\}$ be its trace-class (Saworotnow and Friedell [2]); let $X$ be a locally compact Hausdorff space and let $\mu$ be a positive $A$-valued Borel measure on $X$. The last statement means that $\mu$ is defined on the class $\beta$ of all Borel subsets $\Delta$ of $\mathrm{X}$ having the property that $\Delta \subset Q$ for some compact set $Q$, and $\boldsymbol{\mu}$ is such that $(\boldsymbol{\mu}(\boldsymbol{\Lambda}) \mathbf{x}, \mathbf{x}) \geq 0$ for all $\boldsymbol{Q} \cdot \boldsymbol{\beta}$ and each $x \in A$. Members of $\beta$ will be called bounded Borel sets (a bounded Borel set is a Borel set included in a compact set). Note that the scalar-valued function $m \Delta=\operatorname{tr} \mu \Delta, \Delta \in \beta$, is an ordinary Borel measure on $X$; it coincides with the total variation $|\mu|$ (Definition in 111.1.4 of Dunford and Schwartz [3]) of $\nu$. 
Let $S(X)$ and $S(X, A)$ be respectively the classes of all complex-valued and A-valued simple functions of $x$. One can define the integrals for members $\psi(x)=$ $\Sigma_{i} \lambda_{i} \phi_{\Delta i}(x)$ and $\xi(x)=\Sigma_{i} a_{i} \phi_{\Delta i}(x)\left(\Delta_{i} \epsilon \beta, a_{i} \epsilon A\right.$ and $\lambda_{i}$ 's are complex numbers) of $s(x)$ and $S(X, A)$ in the usual way by setting

$$
\Gamma \psi \mathrm{d} \mu=\Sigma \lambda_{i} \mu \Delta_{i} \text { and } \int \xi \mathrm{d} \mu=\Sigma a_{i} \mu \Delta_{i}
$$

and then extending it to larger classes using the norms

$$
\|\psi\|=\int|\psi| \mathrm{dm}=\Sigma\left|\lambda_{\mathrm{i}}\right| \text { in } \Delta_{\mathrm{i}}
$$

and

$$
\|\xi\|=\Sigma \| a_{i}|| m \Delta_{i}
$$

Let $\mathrm{L}(\mathrm{X})$ and $\mathrm{B}(\mathrm{X}, \mathrm{A})$ denote respectively the classes of those functions to which the integrals are extendable in this fashion. (Note that $S(X)$ is dense in $L(X)$ and $S(X, A)$ is dense in $B(X, A))$.

Then it is easy to see that

$$
r\left(\int \psi \mathrm{d} \mu\right) \leq|| \psi|| \text { and } r\left(\int \xi \mathrm{d} \mu\right) \leq|| \xi||
$$

hold for all $\psi \in L(X)$ and $\xi \in B(X, A)$. (For a discussion of integrals of this type we refer the reader to Bogdanowicz (4]).

LEMMA 1. If aєA and either $\psi \in L(X)$ or $\psi \in B(X, A)$, then $a \psi \in B(X, A)$ and $\int a \psi_{d} \mu=$ $a \int \psi d \mu$. If $\psi \epsilon S(X, A)$ and $\psi \geq 0$-almost everywhere then $\operatorname{tr} \int \psi \mathrm{d} \mu \geq 0$.

PROOF. The first assertion is easy to verify. Let $\psi$ be a simple function such that " $\psi(\mathrm{x}) \geq 0$ " holds ontside of some set $\Delta ; \beta$ with $\mathrm{m} \Delta=\operatorname{tr} \boldsymbol{\mu} \Delta=0$. Then $\psi$ can be represented in the form $\psi=\Sigma_{i=1}^{n} a_{i} \phi_{\Delta i}$ with $\Delta_{1}, \Delta_{2}, \ldots, \Delta_{n}$ disjoint $\left(\Delta_{i} \in \beta\right)$ and $a_{i} \geq 0$ for each $i$ for which " $m \Delta_{i}=r\left(\mu \Delta_{i}\right)=\operatorname{tr}\left(\mu \Delta_{i}\right)>0 "$ holds. Then $\operatorname{tr} \int \psi d \mu=\operatorname{tr} \Sigma_{i} a_{i} \mu \Delta_{i}=\Sigma_{i} \operatorname{tr}\left(a_{i} \mu \Delta_{i}\right)=\Sigma_{i} \operatorname{tr} \sqrt{\mu \Delta_{i}} a_{i} \sqrt{\mu \Delta_{i}} \geq 0$.

Let $L^{2}(\mu)=\left\{\mathrm{f}: \mathrm{X} \rightarrow \mathrm{C} \mid \mathrm{f}\right.$ is m-measurable and $\left.\int|\mathrm{f}|^{2} \mathrm{dm}<\infty\right\}(\mathrm{m}=\mathrm{tr} \mu)$ be the set of all square m-measurable complex-valued functions. Then there is a rA-valued inner product

$$
\left[\psi_{1}, \psi_{2}\right]=\int \bar{\psi}_{1} \psi_{2} \mathrm{~d} \mu
$$

defined on $\mathrm{L}^{2}(\mu)$ such that $\left(\psi_{1}, \psi_{2}\right)=\operatorname{tr}\left[\psi_{2}, \psi_{1}\right]=\int \bar{\psi}_{2} \psi_{1}$ dm is an ordinary scalar product on $\mathrm{L}^{2}(\mu)$ making $\mathrm{L}^{2}(\mu)$ a Hilbert space.

LEMMA 2. Let $\psi_{1}, \psi_{2}, \ldots \psi_{n} \in \mathrm{L}^{2}(\mu)$ and let $a_{1}, a_{2}, \ldots, a_{n} \in A$. Then

$$
\operatorname{tr} \Sigma_{i, j} a_{i}^{\star} \int \bar{\psi}_{i} \psi_{j} d \mu_{a} \geq 0
$$

PROOF. Let $n(\psi)$ denote the norm on $L^{2}(\mu): n(\psi)^{2}=(\psi, \dot{\psi})=\int|\psi|^{2}$ dm. Let $\epsilon>0$ be arbitrary; let $\eta_{1}, \eta_{2}, \ldots \eta_{n} \in S(X)$ be such that $n\left(\psi_{i}-\eta_{i}\right)<\epsilon$ for $i=1,2, \ldots n$. Then

$$
\left|\operatorname{tr} \Sigma_{a_{i}^{*}}^{n} \bar{\psi}_{i} \psi_{j} \mathrm{~d} \mu_{a_{j}}-\operatorname{tr} \Sigma a_{i}^{*} \int_{i}^{\frac{i}{\psi_{j}}} \psi_{j} \mathrm{~d} \mu_{a_{j}}\right|=
$$

$$
\left|\Sigma \operatorname{tr}\left(a_{j} a_{i}^{*} \int\left(\bar{\psi}_{i} \psi_{j}-\bar{\eta}_{i} \eta_{j}\right) d \mu\right)\right| \leq \Sigma r\left(a_{j} a_{i}^{*}\right) r\left(\int\left(\bar{\psi}_{i} \psi_{j}-\bar{\eta}_{i} \eta_{j}\right) d \mu\right) \leq .
$$

$\Sigma|| a_{j}|| \cdot|| a_{i}^{*}|| \int\left|\bar{\psi}_{i} \psi_{j}-\bar{\eta}_{i} \eta_{j}\right| d m \leq \Sigma|| a_{j}|| \cdot|| a_{i}^{*} \|\left(j\left|\bar{\psi}_{i}\right|\left|\psi_{i}-\eta_{j}\right| d m+\int\left|\bar{\psi}_{i}-\bar{\eta}_{i}\right|\left|\eta_{j}\right| d m \leq\right.$

$$
\Sigma\left\|a_{i}\right\| \cdot\left\|a_{j}^{*}\right\|\left(n\left(\psi_{i}\right) \cdot n\left(\psi_{j}-\eta_{j}\right)+n\left(\psi_{i}-\eta_{i}\right) \cdot n\left(\eta_{j}\right)\right) \leq \sum_{i=1} \epsilon\left(2 n\left(\psi_{1}\right)+\epsilon\right)\left\|a_{i}\right\| \cdot\left\|a_{j}^{*}\right\|
$$
and the last sum can be made arbitrarily small by selecting 6 small enough. On the other hand one can see that

$$
\operatorname{tr}\left(\Sigma_{i, j} a_{i}^{\star} \int \bar{\eta}_{i} \eta_{j}{\mathrm{~d} \mu a_{j}}\right)=\operatorname{tr} \int\left(\Sigma_{j} a_{j} \eta_{j}\right)\left(\Sigma_{i} a_{i} \eta_{i}\right) \star d_{\mu} \geq 0
$$


since $\left(\Sigma_{j} a_{j} n_{j}\right)\left(\Sigma_{i} a_{i} \eta_{i}\right) *$ is positive and $\operatorname{simple}_{\star}$. Hence $\operatorname{tr} \Sigma a_{i}^{*} \bar{\iota}_{i} \psi_{j} d \mu a{ }_{j} \geq 0$.

COROLLARY. The expression $z=\Sigma_{i, j}\left(a_{i}^{*} \bar{\psi}_{i} \psi_{j} \mathrm{~d}: a_{j}\right)$ is a positive member of rA.

PROOF. Note that the expression $(z a, a)=\operatorname{tr}\left(a^{\star} z a\right)$ is of the same form as trz. Hence $(z a, a) \geq 0$ for each acA.

Now consider the space $K$ of all tensors $\mathrm{f}=\sum_{\mathrm{i}=1}^{\mathrm{n}} \psi_{\mathrm{i}} \otimes \mathrm{a}_{\mathrm{i}}$ with $\psi_{1}, \psi_{2}, \ldots, \psi_{\mathrm{n}} \in \mathrm{L}^{2}(\mu)$ and $a_{1}, a_{2}, \ldots, a_{n} \in A$. Define the positive form $[f, g]$ on $K$ by setting

$$
[f, g]=\Sigma_{i, j}{ }^{*}\left(\int \bar{\psi}_{i} \eta_{j} d \mu\right) b_{j}
$$

(here $g=\Sigma_{j} \eta_{j} \otimes b_{j}$ ). Let $\eta=\{f \in K:[f, f]=0\}, K^{\prime}=K \mid \eta ;$ we define $L^{2}(\mu) \otimes$ A to be the completion of $K^{\prime}$ with respect to the norm $\|f\|=\sqrt{r[f, f]}$ (modulo the set $\eta$ ). It is not difficult to see that $L^{2}(\mu) \otimes A$ is a Hilbert module.

Let $h$ be a bounded continuous real valued function on $x$. Define the operator $T_{h}$ on $L^{2}(\mu) \otimes$ by setting

$$
T_{h}(f)=T_{h}\left(\Sigma \psi_{i} \otimes a_{i}\right)=\Sigma\left(\psi_{i} h\right) \otimes a_{i}
$$

Then $T_{h}$ is a bounded self-adjoint (in the sense that $\left[T_{h}(f), g\right]=\left[f, T_{h}(g)\right]$ holds). Also $T_{h}$ is A-linear (additive and A-homogeneous in the sense that $T_{h}(f a)=T_{h}(f) a$ for all $\left.f \in L^{2}(\mu) \otimes A, a \in A\right)$.

The fact that $T_{h}$ is hounded (in the sense that " $\left\|T_{h}(f)\right\| \leq M\|f\|$ holds for some M) can be verified directly, using $\$ 10$ of Naimark [5]. Let $f=\Sigma_{i} \psi_{i} \otimes_{i}$ be a fixed member of $K$. Consider the positive linear functional

$$
p(y)=\operatorname{tr}[\mathrm{f}, \mathrm{Ty}(\mathrm{f})]=\operatorname{tr} \sum \mathrm{a}_{i}^{*} \bar{\psi}_{\mathbf{i}} \mathrm{y} \psi_{\mathbf{j}} \mathrm{d} \boldsymbol{\mu}_{\mathrm{j}}
$$

on the space $B C(X)$ of all bounded continuous (complex) functions on $X$. It follows from the proposition I in subsection 4 of $\left\{10\right.$ in Naimark [5] that $p\left(h{ }^{\star} h\right) \leq$ $\|h * h\|_{\infty} p(e)=|| h||_{\infty}^{2} p(e)$. Thus:

$$
\begin{gathered}
\left.|| T_{h}(f)\right|^{2}=\operatorname{tr}\left[T_{h}(f), T_{h}(f)\right]=\operatorname{tr}\left[f, T_{h \star h}(f)\right]=p(h \star h) \leq|| h||_{\infty}^{2} p(e)= \\
|| h||_{\infty}^{2} \operatorname{tr}[f, f]=|| h||_{\infty}^{2}|| f||^{2} .
\end{gathered}
$$

We also see that $\left\|T_{h} \mid \leq\right\| h \|_{\infty}^{\infty}$ It turns out that each bounded self-adjoint A-linear operator is of the form $\mathrm{T}_{h}$ described above.

\section{MAIN RESULTS.}

Definition. An A-linear operator $T$ on a Hilbert module $H$ is said to be cyclic if there exists $f_{o} \in H$ such that the $\operatorname{set}\left\{\Sigma_{k=0}^{n} \lambda_{k} T^{k}\left(f_{o}\right) a_{k}: a_{k} \in A, \lambda_{k} \text { complex }\right\}_{i s}$ dense in $H$ (we assume that $\mathrm{T}^{\mathrm{O}}\left(\mathrm{f}_{\mathrm{o}}\right)=$ If $\mathrm{f}_{\mathrm{O}}=\mathrm{f}_{\mathrm{o}}$ ).

THEOREM 1. For each bounded A-linear self-adjoint operator $T$ on a Hilbert module $H$ there exists a locally compact Hausdorff space $X$, a $r A$-valued positive regular measure $\mu$ defined on the class $\beta$ of bounded (dominated by compact sets) Borel subsets of $X$ and a bounded continuous real valued function $h$ on $X$ such that $H$ is isometrically isomorphic to $\mathrm{L}^{2}(\mu) \mathrm{A}$ and $\mathrm{T}$ corresponds to the operator $\mathrm{T}_{\mathrm{h}}$ (described above) acting on $L^{2}(\mu) \otimes A$. If $T$ is cyclic, then $X$ is homeomorphic to the compact subset of the real line.

PROOF. Let $B$ be the commutative $B^{*}$-algebra generated by $T$ and the identity operator I (note that each member of $B$ is A-linear). Letmbe the set of maximal ideals of $B$, let $r$ be the standard Gelfand topology on $M$ and let $S \longrightarrow S(M)$ be the Gelfand map of $B$ into the continuous complex functions on $m$. Note that $m$ is homeomorphic to the spectrum of $T$, which is a compact subset of the real line. We consider 2 cases. 
CASE I. First assume that there exists $f_{0} H$ such that the set

$$
H^{l}=\left\{\Sigma_{i=1}^{n} s_{i}\left(f_{0}\right) a_{i}: S_{i} \in B, a_{i} \in A\right\}
$$

is dense in $H$ (this is equivalent to the statement that $T$ is cyclic).

Let $\beta$ be the class of all Borel subsets of $m$ (each $\Delta \cdot \beta$ is bounded since $m$ is compact) and let $\Delta \rightarrow{ }_{\Delta}$ be a spectral measure on $\beta(\xi 17$, Proposition II in subsection 4 of Nainark [5]) such that $S=\int_{m} S(M) d_{M^{*}}$ Note that each $P_{\Delta}$ is $A^{-l i n e a r}$ ;ince it commutes with linear maps $f \longrightarrow f a(a \in A)$ (which commute with all SeB). Then map

$$
\Delta \rightarrow \mu_{\Delta}=\left[\mathrm{f}_{0}, \mathrm{P}_{\Delta} \mathrm{f}_{0}\right]
$$

is a rA-valued positive measure on $\beta$, and for each $S \in B$ we have

$$
\int_{M} \mathrm{~S}(M) \mathrm{d} \boldsymbol{\mu}(\mathrm{M})=\int \mathrm{S}(\mathrm{M}) \mathrm{d}\left[\mathrm{f}_{0}, \mathrm{P}_{M} \mathrm{f}_{0}\right]=\left[\mathrm{f}_{0}, \int \mathrm{S}(\mathrm{M}) \mathrm{dP}_{M}\right]=\left[\mathrm{f}_{0}, \mathrm{Sf}_{0}\right]
$$

(here, as above, [ ] denotes the generalized inner product on $H$ ). In this case we can take $\mathrm{x}=m$. The correspondence

$$
\mathrm{Sf}_{\mathrm{o}} \longleftrightarrow \mathrm{S}(\mathrm{M})
$$

is a (linear) isomorphism between the linear subspace $K=\left\{S_{f o} \mid S \in B\right\}$ of $H$ and $C(X)=$ $c(m)$. This correspondence can be extended in the obvious way to the isomorphism between the closure of $K$ and the Hilbert space $L^{2}(\mu)$. The $r A$-valued inner product is also preserved by this correspondence: if $S_{1}, S_{2} \in B$ then

$$
\left[\mathrm{s}_{1} \mathrm{f}_{0}, \mathrm{~S}_{2} \mathrm{f}_{\mathrm{o}}\right]=\left[\mathrm{f}_{0}, \mathrm{~s}_{1}^{*} \mathrm{~S}_{2} \mathrm{f}_{\mathrm{o}}\right]=\int \overline{\mathrm{S}}_{1}(\mathrm{M}) \mathrm{S}_{2}(\mathrm{M}) \mathrm{d} \boldsymbol{\mu}(\mathrm{M})
$$

We extend this isomorphism to a correspondence between $\mathrm{H}^{\mathrm{l}}$ and a dense subset of $L^{2}(\mu) \otimes A$ by setting

$$
\Sigma_{k} S_{k}\left(f_{o}\right) a_{k} \longleftrightarrow \Sigma S_{k}(M) \otimes a_{k}
$$

This correspondence also preserves the (vector) inner product: if $f=\Sigma s_{k}\left(f_{o}\right) a_{k}$ and $g=\Sigma Q_{i}\left(f_{o}\right) b_{i}$, then

$$
[f, g]=\Sigma_{k, i} a_{k}^{*}\left[s_{k}\left(f_{o}\right), Q_{i}\left(f_{o}\right)\right] b_{i}=\Sigma_{k, i} a_{k}^{*} \int \bar{s}_{k}(M) Q_{i}(M) d \mu b_{i}
$$

We extend it to an isomorphism between $H$ and $L^{2}(\mu) \otimes A$. It is easy to check that $T$ correponds to the operator $T_{h}$ of multiplication with function $h(M)=T(M)$ :

$$
T\left(\Sigma_{k} S_{k}\left(f_{o}\right) a_{k}=\Sigma_{k} T S_{k}\left(f_{o}\right) a_{k} \longleftrightarrow \Sigma_{k} T(M) S_{k}(M) \otimes a_{k}\right.
$$

The function $h$ is real valued since $T^{\star}=T$, and ||$h||_{\infty} \leq|| T||$.

Note also that in this case $M$ is homeomorphic to the spectrum of $T$, which is a compact subset of the real line. This implies the last assertion of the theorem.

CASE II. Now let us consider the general case. For any $f(H$ let $H(f)$ be the closure of the set $\left\{\sum_{i=1}^{n} S_{i}(f) a_{i}: S_{i} \in B, a_{i} \in A\right\}$. Then it follows from Lemma 2 in Saworotnow [6] that $f \in H(f)$. Also both $H(f)$ and its orthogonal complement $H(f)$ (which coincides with the set $H(f)^{p}=\{g \in H:[g, h]=0$ for all h€H(f) $\}$ (Lemma 3 of Saworotnow [6])) are invariant under $\mathrm{T}$.

It follows from this fact and Zorn's Principle that there exists a set $\left\{\mathrm{f}_{y}: y \in \Gamma\right\}$ of mutually orthogonal members of $\mathrm{H}$ such that $\mathrm{H}=\Sigma_{\gamma} \otimes H\left(\mathrm{f}_{y}\right), \mathrm{H}\left(\mathrm{f}_{\gamma}\right) \perp \mathrm{H}\left(\mathrm{f}_{\beta}\right)$ if $\gamma \neq \beta$, and each $H\left(\mathrm{f}_{\gamma}\right)$ is invariant under $\mathrm{T}$.

For each $\gamma \in \Gamma$ and $S \in B$ let $s_{\gamma}$ be the restriction of $S$ to $H\left(f_{\gamma}\right)$, and let $B \gamma=$ $\left\{S_{\gamma}: S \in B\right\}$. It follows from part I (case I) of this proof that for each $\gamma \in \Gamma$ there exists a compact Hausdorff space $\left(m_{\gamma},{ }^{r} \gamma\right)$, a rA-valued positive Borel measure $\vdash^{\prime} \gamma$ and 
a continuous real valued function $h_{y}()$ on $m_{y}$ such that $\mathrm{H}\left(\mathrm{f}_{\gamma}\right)$ is isomorphic to $L^{2}\left(\mu_{\gamma}\right) A$ and action of the operator $T_{\gamma}$ (the restriction of $\mathrm{T}$ ) corresponds to the multiplication with $h_{y}$ on $L^{2}\left(\mu_{\gamma}\right)$. Note also that $h_{\gamma}(M) \leq \| \mathrm{T}||$ for each $M \in m_{\gamma}$.

Let $\mathrm{X}=\mathrm{U} m_{\gamma}$ and let $r$ be the topology on $\mathrm{X}$ defined by the requirement that a set $O C X$ is open (Ot+) if and only if $o \cap m \gamma$ belongs to r $\gamma$ for each $\gamma \in \Gamma$. Let $\beta$ be the class of all bounded Borel subsets of $x$. For each $\Delta \epsilon \beta$ there are indices (we use a ,implified notation here) $1,2, \ldots, n \in \Gamma$ such that $\Delta c \bigcup_{i=1}^{n} m_{i}$. We set

$$
\mu(\Delta)=\sum_{i=1}^{n} \mu_{i}\left(\Delta n m_{i}\right)
$$

Then $\beta$ is a ring and $\boldsymbol{\mu}$ is a positive $r$-valued measure on $\beta$. We define the function $h$ on $X$ by setting $h(M)=h_{\gamma}(M)$ where $\gamma \in \Gamma$ is such that $M \in m_{\gamma}$. Then it is easy to see that $h$ has the required properties.

To complete the proof it is now sufficient to show that $L^{2}(\boldsymbol{\mu}) \otimes A=\Sigma_{\gamma} L^{2}\left(\mu_{\gamma}\right) \otimes A$. First note that each $L^{2}\left(\mu_{\gamma}\right)$ is included in $L^{2}(\mu)$ and that $L^{2}(\mu)=\Sigma_{\gamma} L^{2}\left(\mu_{\gamma}\right)$ (easy to verify). Now let $f \in \mathrm{L}^{2}(\mu) \otimes A$. For each $\epsilon>0$ one can find $g=\Sigma_{i=1}^{\prime \prime} \psi_{i} \otimes a_{i}$ such that || $\mathrm{f}-\mathrm{g}||<\epsilon$ with $\psi_{\mathrm{i}} \mathrm{L}^{2}(\boldsymbol{\mu})$. But each $\psi_{\mathrm{i}}$ can be approximated in $\mathrm{L}^{2}(\boldsymbol{\mu})$ by wxpressions of the form $\Sigma_{j=1}^{n} \phi_{j}$ with $\phi_{j} \in L_{L}^{2}\left(\mu_{\gamma_{j}}\right)$ for some $\gamma_{1}, \gamma_{2}, \ldots, \gamma_{n} \epsilon \prod_{2}$ Thus $\mathrm{f}$ can be approximated (as close as we please) by members $\Sigma_{i=1}^{n}\left(\Sigma_{j} \phi_{j}\right) a_{i}$ of $\Sigma_{\gamma} L^{2}\left(\mu_{\gamma}\right) A$, i.e., $g$ is a ineinber of $\Sigma_{\gamma} L^{2}\left(\mu_{\gamma}\right)$ A.

Conversely, let $\mathrm{f} \in \boldsymbol{\Sigma}_{\boldsymbol{y}} \mathrm{L}^{2}\left(\boldsymbol{\mu}_{\boldsymbol{y}}\right) \otimes \mathrm{A}$; then $\mathrm{f}$ can be approximated by finite sums of expressions of the type $\sum_{i=1}^{n} \psi_{i} \otimes a_{i}$ with $a_{i} \in A$ and $\psi_{1}, \psi_{2}, \ldots \psi_{n}$ belonging to some $\mathrm{I}^{2}(\mu \beta)$ with $\beta \epsilon \Gamma$. We may conclude that $f \in \mathrm{L}^{2}(\mu) \mathrm{A}$ since $\mathrm{L}^{2}\left(\mu_{\gamma}\right) \subset \mathrm{L}^{2}(\mu)$ for each $\gamma^{\prime}$. The reader should be able to give a precise argument here.

THEOREM 2. Let $Z$ be a family of bounded A-linear operators on a Hilbert module H (over an $H^{*}$-algebra A) such that each member of $Z$ and its adjoint (with respect to the generalized inner product) commute with any other member of 7 . In particular, Z could be a commutative *-algebra of A-linear operators on $\mathrm{H}$. Then there exists a locally compact Hausdorff space $X$, a $r A$-valued positive Borel measure $\boldsymbol{\mu}$ on $X$ and a map $\mathrm{T} \rightarrow \mathrm{h}_{\mathrm{T}}$ of $\mathrm{Z}$ into complex valued functions on $\mathrm{X}$ such that $\mathrm{H}$ is isomorphic to $\mathrm{L}^{2}(\boldsymbol{\mu}) \mathrm{A}$ and each $\mathrm{T}$ corresponds to multiplication with some function $\mathrm{h}_{\mathrm{T}}$. Moreoever $\left\|\mathrm{h}_{\mathrm{T}}\right\|_{\infty} \leq$
$\|\mathrm{T}\|$ for each $\mathrm{T} \epsilon \%$.

PROOF. The proof is essentially the same as the proof of Theorem 1 above. We use the *-algebra of operators generated by $Z$ (and the identity operator I) instead of the algebra generated by the operator $\tau$ (and $I$ ).

COROLLARY 1. Each *-representation of a commutative *-algebra by bounded A-linear operators is of the form $x \rightarrow T_{h}$, where $T_{h}$ is an operator of multiplication with a complex valued function $h=h_{x}$ described before Theorem 1 .

This corollary could be considered as a generalization of Theorem 65 in Mackey [7] if we disregard the fact that Mackey considers more general (self-adjoint) algebras and we do not specify the space $x$ on which the functions $h=h_{x}$ act (also our Hilbert module does not have to be separable (as a Hilbert space)).

COROLLARY 2. Let $G$ be a commutative locally compact group with composition +

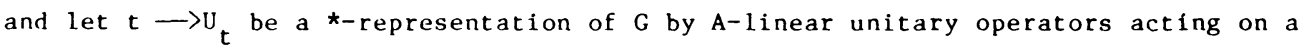
Hilbert module $\mathrm{H}$. Assume that there exists a vector $\mathrm{f}_{0} \mathrm{H}_{\mathrm{H}}$ such that the submodule $\mathrm{H}_{0}$, generated by the vectors of the form $U_{t}\left(f_{0}\right)$, is dense in $H$. Then there exists a compact Hausdorff space $\mathbb{Z}$, a positive $r A-v a l u e d$ Borel measure $\mu$ on $M$ and a map 
$t \rightarrow g_{t}$ of $G$ into the continuous functions on $M$ such that $H$ is (isometrically) isomorphic to $L^{2}(\mu) A$ and each $U_{t}$ corresponds to multiplication members of $L^{2}(\mu)$ with $g_{t} \cdot$

The map $t \rightarrow g_{t}$ has the following properties (for edch $t \in G$ and all Mats:

$$
\begin{aligned}
& g_{0}(M)=1 \text { (here } 0 \text { is the identify of } G \text { ) } \\
& \left|g_{t}(M)\right|=1 \\
& g_{-t}(M)=\bar{g}_{t}(M) \\
& g_{t+s}(M)=g_{t}(M) g_{s}(M)
\end{aligned}
$$

It is appropriate at this point to mention a certain application of the last corollary. Let $\mathrm{G}, \mathrm{A}$ and $\mathrm{H}$ be as above, and let $\xi: G \longrightarrow \mathrm{H}$ be a generalized stationary process (Saworotnow $[8]$ ), i.e., $\xi$ is an H-valued function on $G$ such that $(\xi(t+r), \xi(s+r))=(\xi(t), \xi(s))$ for all $t, r, s \in G$. Let ${ }^{H} \xi$ be the submodule generated by the vectors of the form $\xi(t), t \in G\left(H_{\xi}=\right.$ closure of $\left.\left\{\tilde{\Sigma}_{k=1}^{n} \xi\left(t_{k}\right) a_{k}: t k G\right\}\right)$.

For each teG consider the operator $U_{t}$ on $H_{\xi}$ defined by

$$
U_{t}\left(\Sigma_{k=1}^{n} \xi\left(t_{k}\right) a_{k}\right)=\Sigma_{k=1}^{n} ;\left(t_{k}+t\right) a_{k} \text { and let } f_{0}=\xi(0) \text {. }
$$

Then the map $t \rightarrow U_{t}$ is a representation of $;$ by A-linear unitary operators and $i t$ is easy to see that the assumptions of Corollary 2 are fulfilled. Let $\mathbb{M}^{\mu} \mu$ and $\xi_{t}$ he as in Corollary 2 and let $\mathrm{f}(\mathrm{M})$ be the member of $\mathrm{C}(\boldsymbol{m})$ corresponding $\left.\mathrm{to}_{0}=\boldsymbol{\xi}(1)\right)$. Then the space ${ }^{H} \xi$ is isomorphic to $L^{2}(\mu) A$ and each $U_{t}$ corresponds to multiplication of members of $L^{2}(\boldsymbol{\mu})$ with $g_{t}$. For each teG let $h_{t}(M)=g_{t}(M) f(M)$. In this fashion we Arrived at a concrete representation of the abstract stationary process $\xi$ by the

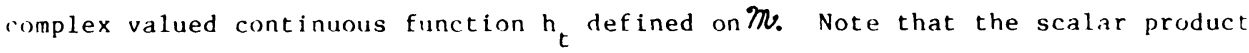
$(\xi(t), \xi(s))$ corresponds to the expression

$$
\begin{aligned}
& \int h_{t}(M) \overline{h_{s}(M)} \mathrm{d} \mu(M)=\int g_{t}(M) \overline{g_{s}(M)} f(M) \overline{f(M)} \mathrm{d} \mu(M)= \\
& \int g_{t}(M) g_{\sim s}(M)|f(M)|^{2} \mathrm{~d} \mu(M)=\int g_{t-s}(M)|f(M)|^{2} \mathrm{~d} \mu(M)
\end{aligned}
$$

and this expression depends on $t-s$ only and is independent of a particular choice of $t$ and $s$.

\section{CONCLUDING REMARK.}

To conclude the paper we make the following remark about the operator $T_{h}$ discussed above. It is easy to see that we do not need at all to assume existence of a (locally compact) topology on the space $X$ (discussed at the beginning of this paper). Let $\mu$ be a positive $r A$-valued measure defined on some $\sigma$-ring of subsets of $X$. If $h$ is any $\operatorname{tr} \mu$-measurable essentially bounded real valued function on $X$ then the corresponding operator $\mathrm{T}_{\mathrm{h}}$ on $\mathrm{L}^{2}(\mu) \otimes \mathrm{A}$,

$$
T_{h}\left(\Sigma_{i} \psi_{i} \otimes a_{i}\right)=\Sigma_{i}\left(\psi_{i} h\right) \otimes a_{i}
$$

is also self-adjoint, A-linear and bounded. The fact that $T_{h}$ is bounded can be verified in the same way as above using the algebra $B$ of all essentially bounded tr $\mu$-measurable complex-valued functions on $\mathrm{X}$. 


\section{REFERENCES}

1. AMBROSE, W. Structure Theorem for a Special Class of Banach Algebras, Trans. Amer. Math. Soc. $57(1945), 364-386$.

2. SAWOROTNOW, P.P. and FRIEDELL, J.C. Trace-class for an Arbitrary H*-algebra, Proc. Amer. Math. Soc. 26 (1970), 95-100.

3. DUNFORD, N. and SCHWARTZ, J. Linear Operators, Part 1, Interscience, New York, 1958.

4. BOGDANOWIC\%, W.M. A Generalization of the Lebesgue-Bochner-Stieltjes Integral and the New Ap!roach to the Theory of Intergration, Proc. of the National Academy of Sciences 53 (1965), 492-498.

5. NALMAKK, M.A. Normed Rings, Moscow, 1968.

6. SAWOROTNOW, P.P. A Generalized Hilbert Space, Duke Math. Journal 35 (1968), 191-197.

7. MACKEY, G.W. Commutative Banach Algebras, Lecture Notes - 1952, Harvard University, Livraria Castelo, Rio de Janiero, 1959.

8. SAWOROTNOW, P.P. Abstract Statjonary Processes, Proc. Amer. Math. Soc. 40 (1973), 585-589.

9. HALMOS, P.R. Measure Theory, Van Nostrand, New York, 1950.

l). ROBERTSON, A.P. and ROBERTSON, W.J. Topological Vector Spaces, Cambridge University Press, 1966.

11. SAWOROTNOW, P.P. On a Realization of a Complemented Algebra, Proc. Amer. Math. Soc. $15(1964), 964-966$.

12. SAWOROTNOW, P.P. Linear Spaces with an $H^{*-a l g e b r a ~ V a l u e d ~ I n n e r ~ P r o d u c t, ~ T r a n s . ~}$ Amer. Math. Soc. 262 (1980), 543-549. 


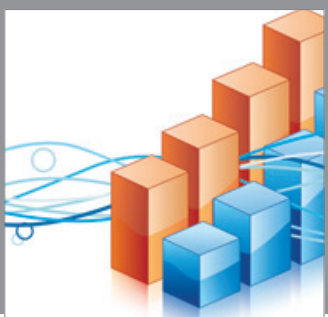

Advances in

Operations Research

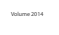

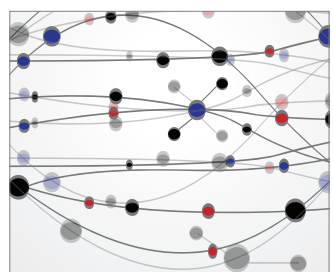

\section{The Scientific} World Journal
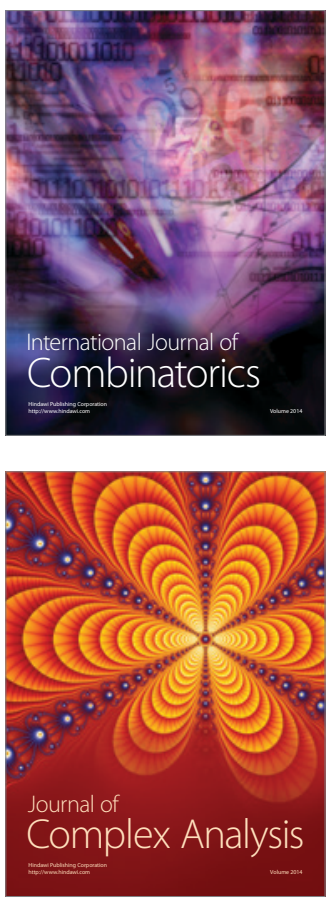

International Journal of

Mathematics and

Mathematical

Sciences
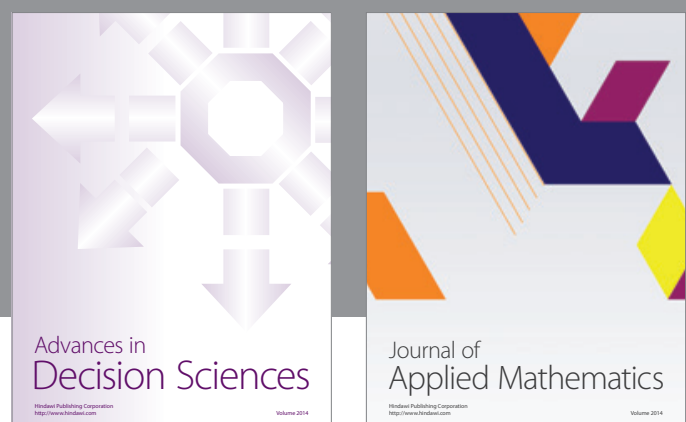

Journal of

Applied Mathematics
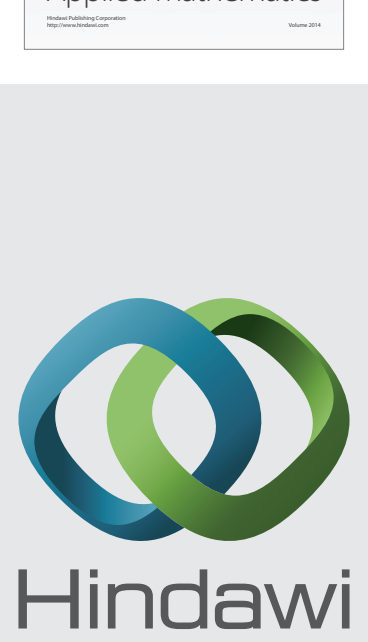

Submit your manuscripts at http://www.hindawi.com
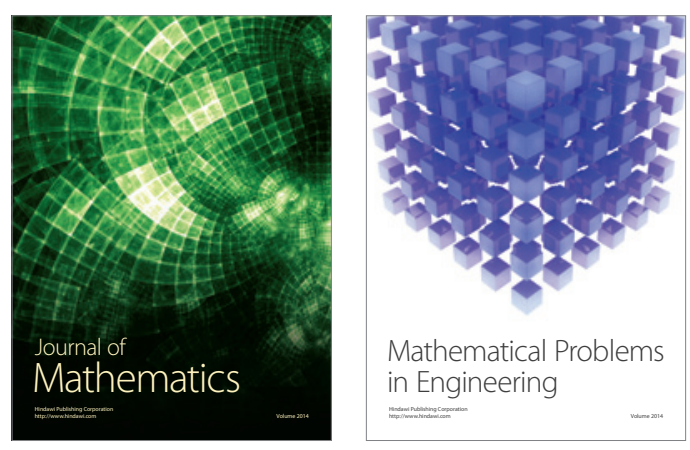

Mathematical Problems in Engineering
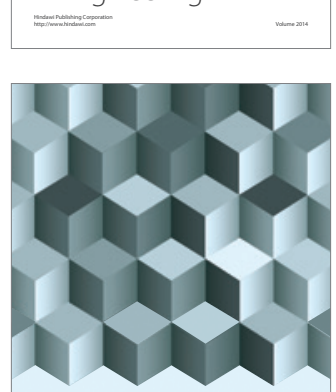

Journal of

Function Spaces
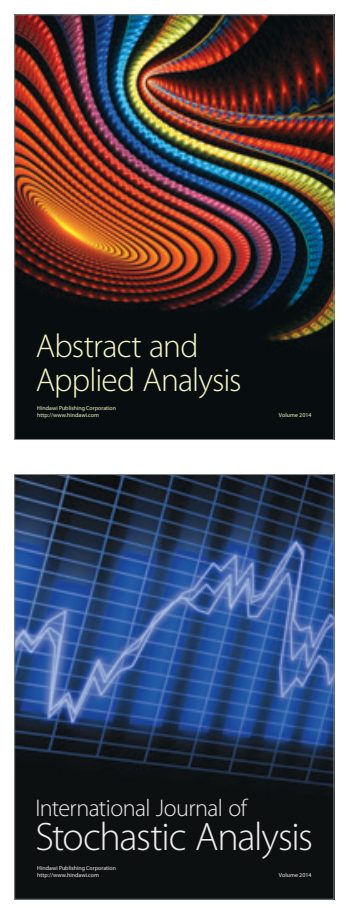

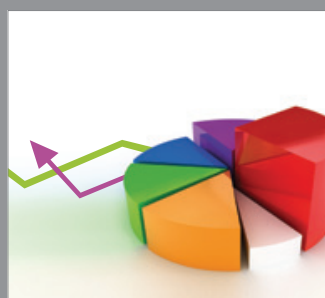

ournal of

Probability and Statistics

Promensencen
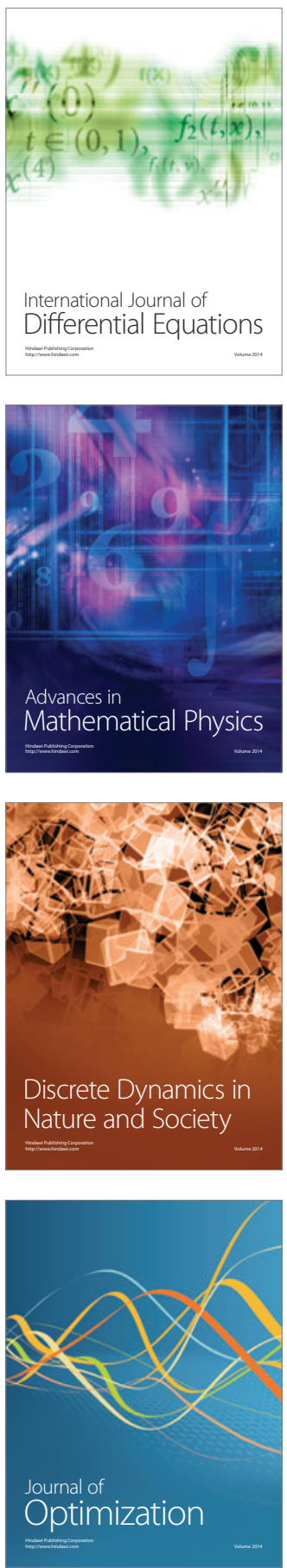